\title{
Operational Engineering of the COLUMBUS Thermal and Environmental Control System: Achievements, Optimizations
}

\author{
Andreas O. Kohlhase and Norbert Porth* \\ German Space Operations Center, DLR Oberpfaffenhofen, 82230 Wessling, Germany \\ Julian Doyé ${ }^{\dagger}$ \\ German Space Operations Center, LSE Space Engineering 83 Operations AG, 82230 Wessling, Germany
}

\begin{abstract}
After commissioning of the European space-borne science laboratory Columbus, many operational products had to be improved and adapted to changing environmental conditions and new operational experiences. In this paper, we focus on the operational engineering of the Thermal Control as well as on the Environmental Control and Live Support System which are mainly influenced by crew activities, payloads and systems. We present an anomaly handling process how to overcome unexpected anomalies or system change requests. We apply this approach to two dedicated examples for which operational workarounds, a final recovery procedure and even a new operations concept had to be developed: (1) The introduction of a so-called Low Condensing Mode for the condensate heat exchanger due to changing station wide requirements and (2) impacts of unexpected smoke detector contamination. With the help of telemetry data, we explain workaround, final solution and operations concept development. We conclude how to change the Columbus air-conditioning and thermal control design to improve humidity control, condensate collection and smoke detector performance and give recommendations for the air-conditioning and smoke detector design of future human space flight missions.
\end{abstract}

\section{Nomenclature}

$\dot{Q}_{M T H X}, \dot{Q}_{L T H X}=$ heat flux in the space station medium and low temperature interface heat exchanger

$\dot{Q}_{I F H X} \quad=$ heat flux in both space station interface heat exchangers

$\dot{Q}_{C H X}, \dot{Q}_{\text {Plenum }}=$ heat flux in the Columbus condensate heat exchanger and plenum

$\Delta p \quad=$ Measured difference water pressure over the plenum

$c_{p} \quad=$ Specific heat capacity of water

$\dot{m}_{I F H X}, \dot{m}_{t o t} \quad=$ Water mass flow in the interface heat exchanger line and total loop mass flow

$\mu \quad=$ Ratio of total to interface heat exchanger line mass flow

$\Delta T_{M T}, \Delta T_{L T} \quad=$ Measured difference temperature in the medium and low interface heat exchanger

$T_{W P A} \quad=$ Water temperature reading in the Columbus water pump assembly

$T_{W T S B 6} \quad=$ Water temperature reading in the Columbus wet temperature sensor block 6

$\vartheta, \vartheta_{d} \quad=$ Cabin air and dew point temperature

$\varphi \quad=$ Relative air humidity

$U_{\text {Obsc. }}, U_{S c .} \quad=$ Smoke detector obscuration and scatter voltage readings

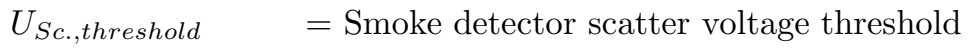

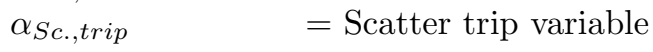

$\tau_{\text {trip }} \quad=$ Percent trip

${ }^{*}$ COL Systems Engineer \& COL Flight Director, Mission Operations, DLR Oberpfaffenhofen, 82230 Wessling, Germany.

${ }^{\dagger}$ COL Systems Lead, Mission Operations, DLR Oberpfaffenhofen, 82230 Wessling, Germany. 


\section{Introduction}

$\mathrm{T}$ He Columbus module is the biggest contribution of the European Space Agency ESA to the International Space Station ISS. On February 11, 2008, four days after its launch from Kennedy Space Center, the laboratory was successfully attached to Node 2 (Harmony module) of the ISS. The purpose of Columbus is to serve as a unique platform for different fields of research: Human physiology, biology, fundamental physics, material sciences and fluid physics. Furthermore, external experiment facilities allow the long-term and non-perturbed observation of the Earth and the universe. Main contractor for Columbus is EADS Space Transportation in Bremen where it was finally assembled. The supporting elements, however, were delivered by Thales Alenia Space in Turin. The European laboratory is operated by the Columbus Control Center at the German Space Operations Center nearby Munich.

On flight day 5 of STS-122/1E, the stations's robotic arm moved CoLumBus from the shuttle's cargo bay to the starboard site of Node 2 to which it was attached. This activity was supported by two astronauts during the first Extra Vehicular Activity (EVA) of the mission to connect Columbus mechanically to Node 2. After this initial installation, the CoLumBus flight control team run a system procedure to finally activate essential equipment like the power distribution, thermal control, data management and communication system. The activation of the essential systems allowed the crew to work in the laboratory and to reconfigure system equipment and payload racks from launch to nominal configuration while ground continued to commission the remaining systems. On flight day 9, the third EVA was performed to attach two external experiment facilities to the external payload facility. Two days later, the Columbus commissioning phase was accomplished and the laboratory was ready for nominal operations. The external and internal configuration of the module is illustrated by figure 1 .

During the first months of operations, system equipment was checked to analyze system behavior and to improve performance and first experiments were run. Maintenance tasks like the dry-out of the

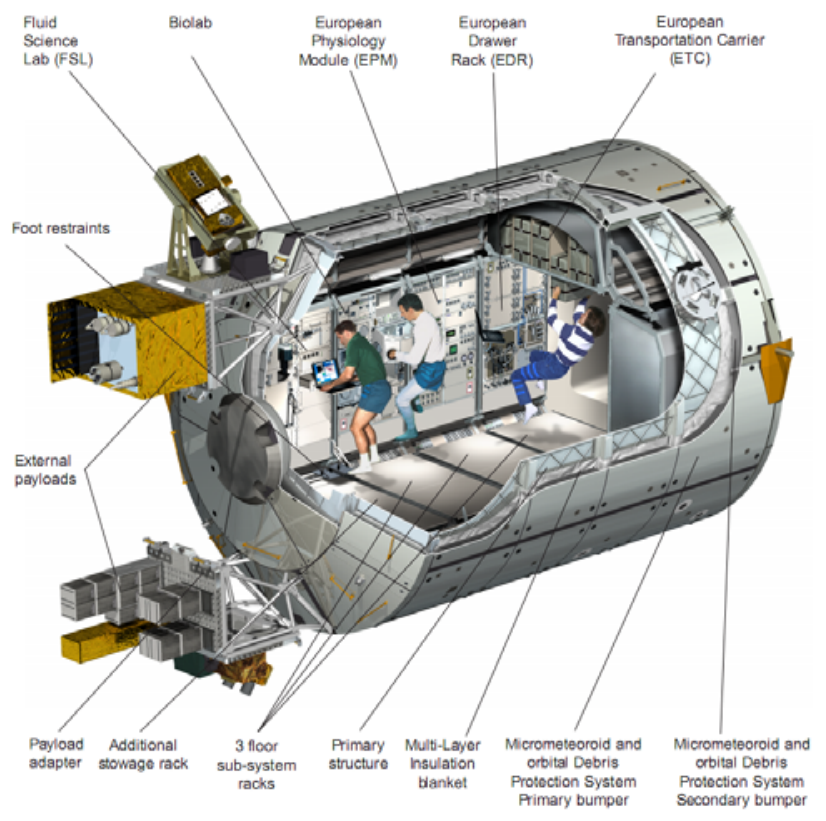

Figure 1. Columbus external and internal configuration. Top is Overhead, bottom Deck, the front side Forward and the back side Aft. The left side is Starboard Cone and the right one Port Cone with the interface to Node 2. condensate heat exchanger was performed which allowed further analysis of system behavior. On the other hand, these activities revealed non-optimized flight procedures and telemetry display pages. As a consequence, those products had to be revised and re-validated during nominal operations. Furthermore, non-analyzed system behavior and unexpected events manifested limitations in the operations concepts and products which triggered the development of workarounds, recovery procedures and new concepts. This became especially evident by the influence of crew and systems on the Thermal Control (TCS) and Environmental Control \& Live Support System (ECLSS).

In the early summer of 2008, station-wide humidity conditions suddenly changed which required to stop condensate collection in Columbus. However, the Columbus heat exchanger was not designed for non-condensing. The developed workaround and final solution was to increase the heat exchanger inlet temperature by $2^{\circ} \mathrm{C}$ which reduced the condensate collection sufficiently to account for the changing requirements. This was declared as a new operational CoLumbus ECLSS mode, the so-called Low Condensing Mode. At the end of 2008, an sudden increase of smoke detector 2 lens contamination was observed. The optical contamination behavior was investigated by changing airloop configurations, however, no improvement of performance was observed. An extensive crew and ground procedure was developed to access and clean smoke detector 2. To save time for final recovery procedure development an intermodule fan was switchedoff to mitigate contamination effects. Finally, in late summer 2009 the crew could successfully clean smoke detector 2 . 


\section{Thermal Control System}

$\mathrm{T}$ HE task of a thermal control system is essentially to ensure that the temperatures of all mechanical, electric and electronic units of a spacecraft are within specified operating temperature ranges during all mission phases. ${ }^{1}$ The Columbus thermal system can be divided in two main parts, the passive thermal system and the active thermal system. The active part consists of a circulating water loop with an interface to the United States Orbit Segment (USOS) with the capability to collect the heat loads in Columbus and reject them to the ISS external cooling loops. The passive system instead consists of various kinds of insulation material, blankets and heater elements.

\section{A. Passive TCS}

In order to reduce the heat flux from and to the internal CoLumbus environment and to minimize temperature fluctuations under varying external radiation environments two different Multi-Layer Insulations (MLI) blankets are used to insulate the shell structure. The blankets are mounted beneath the Micrometeorid \& Debris Protection System (MDPS) to the primary shell structure itself (see fig. 1). The external visible layer of MLI type A consists of a silver-coated Kapton foil with medium absorptance and emission properties. It is used to cover the Columbus cylinder surface as well as port and starboard cones and mainly minimizes a heat leak during eclipses. MLI type B consists of beta cloth with lower absorptance and higher emission properties which is preferred as external layer for the MLIs that are partially or directly exposed to space. Type B is used to cover, e.g., rings and longerons. Figure 2 shows a part of the primary shell structure insulated with both MLI types.

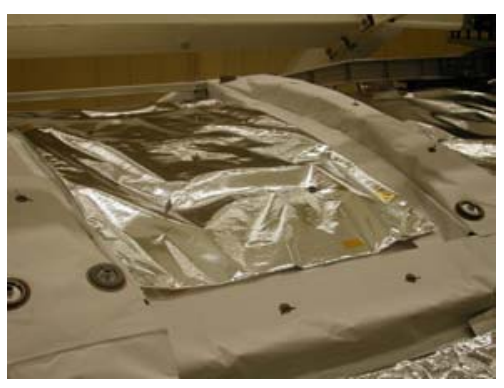

Figure 2. Primary shell structure insulated with type $A$ and type $B$ MLIs.

Along with the shell insulation, foam material is used to insulate pipings and other components. Assemblies in the low temperature section of the active thermal fluid loop can reach temperatures down to $1^{\circ} \mathrm{C}$. In order to thermally isolate these assemblies and to limit humidity flow through the material these assemblies are covered with special, anti-expanding foam layer insulations. To prevent condensation on the internal shell the Passive TCS provides heater elements and temperature sensors on the external shell. The heaters are grouped to six-heater circuits with three thermistors and thirteen heater elements for each circuit. Six circuits belong to one heater chain that is powered and controlled by one Heater Control Unit (HCU). Two chains are implemented for redundancy purposes and nominally only one heater chain is on control. In the event of cabin depressurization dedicated heaters are used to prevent freezing of the Cabin Depressurization Assembly (CDA) vents.

\section{B. Active TCS}

Apart from the space radiation environment, internal head loads have to be dissipated to avoid a heating of the module. Heat loads are electrical equipment on system and payload level as well as the astronauts themselves who transfer body heat into the air by radiation. Since the heat release through the shell is very limited an active cooling system must be applied to dissipate the remaining energy. This is realized by an internal TCS single water-loop that collects and transports dissipative heat to two Heat Exchangers (HX) outside of Node 2 that provide heat rejection capability to the USOS external TCS loops A and B. The overall cooling loop architecture with the Common Berthing Mechanism (CBM) is shown in figure 3 .

The Columbus internal TCS consists of a low temperature section $\left(4-6^{\circ} \mathrm{C}\right)$ and a medium temperature section $\left(16-18^{\circ} \mathrm{C}\right)$. A Water Pump Assembly (WPA) pumps the warm water $\left(\approx 20^{\circ} \mathrm{C}\right)$ to the Medium Temperature HX (MTHX) where it is cooled down to $\approx 12^{\circ} \mathrm{C}$. Then the water temperature reaches the low level of $5^{\circ} \mathrm{C}$ after having passed the Low Temperature HX (LTHX). In the Condensate Heat Exchanger (CHX) the heat from the air is exchanged with coolant. System cold plates on which the most electrical units are installed collect their dissipative heat through heat conduction. In ten parallel water lines further heat is collected that dissipates from the International Standard Payload Racks (ISPR).

Figure 4 illustrates the water loop control mechanism of the Columbus active TCS. Since the TCS is absolutely vital, the system is accordingly redundantly designed. Three active water loop control laws are applied to regulate plenum $\Delta p$, CHX inlet and plenum temperature. 
The water flow and pressure control in the water loop is based on a plenum $\Delta p$-control, i.e., that the WPA adjusts the pump speed such that the $\Delta p$ over the plenum always remains within a control range of a default set value. For $\Delta p$-control, the readings of the main sensor of Delta Pressure Sensor Blocks (DPSB) 1 and 3 are used. The low temperature section is controlled by the Water Modulating Valve (WMV) 3 that mixes the cold water with the water coming from the warm by-pass. The CHX inlet water temperature is measured with the two sensors of the Wet Temperature Sensor Block (WTSB) 3. The same control mechanism applies to the control of the medium temperature section. WMV 1 mixes the warm bypass water with that coming from the CHX. The plenum temperature is measured by the first two sensors of WTSB 1. To isolate main

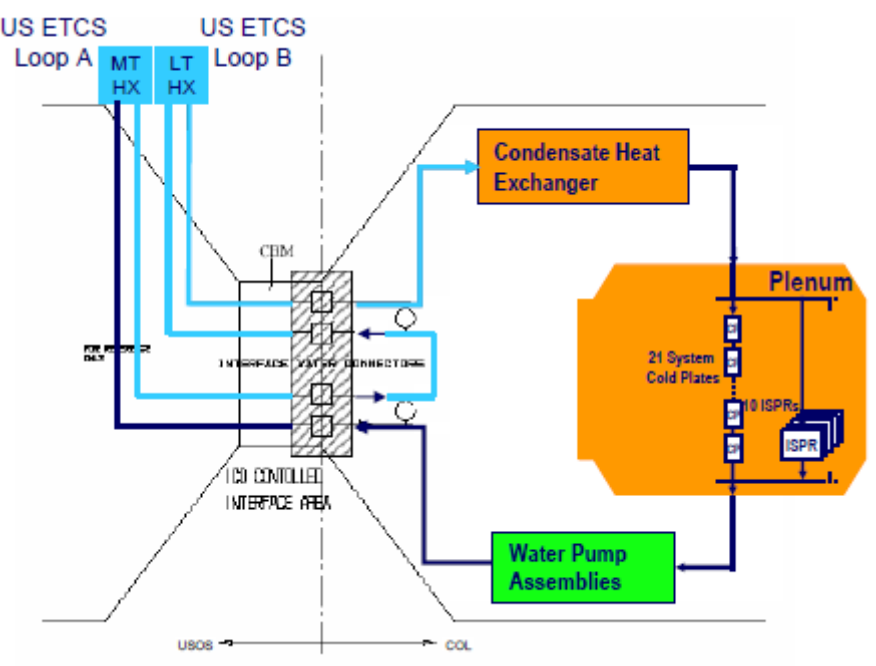

Figure 3. Columbus water cooling loop architecture interfacing with the US External TCS Loops A and B. or bypass lines Water On-Off Valves (WOOV) can be used and to provide the ISPRs with coolant Water Flow Selection Valves (WFSV) must be opened.

Thermodynamically spoken, the amount of heat to be rejected in the ISS Interface HXs (IFHX) can be balanced as

$$
\begin{aligned}
\dot{Q}_{M T H X}+\dot{Q}_{L T H X} & =\dot{Q}_{C H X}+\dot{Q}_{\text {Plenum }} \\
\dot{Q}_{I F H X s} & =c_{p} \dot{m}_{I F H X}\left(\Delta T_{M T}+\Delta T_{L T}\right) \\
& =c_{p} \eta \dot{m}_{t o t}\left(T_{W P A}-T_{W T S B 6}\right)
\end{aligned}
$$

under the assumption of microgravity, no pressure losses and a constant tube diameter. In a real incompressible fluid, pressure losses are caused by inner tube friction. The WPA 1 compensates these energy losses to maintain a constant water mass flow by injecting mechanical energy into the closed water loop. The heat transfer in both IFHXs that is based on conduction can thus be estimated by the specific enthalpy difference $c_{p}\left(T_{W P A}-T_{W T S B 6}\right)$ and the total water mass flow $\dot{m}_{t o t}$ measured in WPA 1 . Equation (1) yields a heat power of $\approx 0.6 \mathrm{~kW}$ in the CHX and $\approx 1.4 \mathrm{~kW}$ in the plenum. Comparing the total amount of $\approx 2 \mathrm{~kW}$ against heat flux telemetry data provided by the US partners proves Eq. (1). ${ }^{2}$

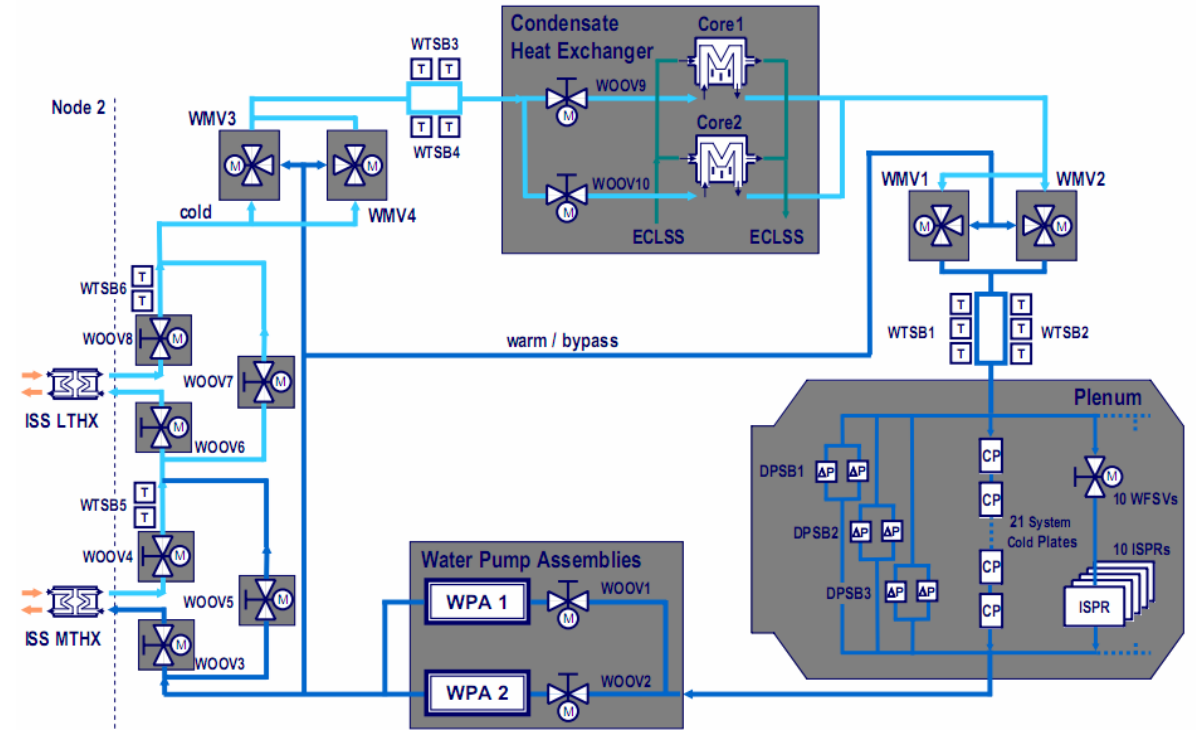

Figure 4. Water Cooling loop control mechanism of the Columbus active TCS. 


\section{Environmental Control and Life Support System}

THE purpose of ECLSS is to assure the biological autonomy of humans in an isolated environment, while 1 creating a physiologically acceptable environment which makes residence there comfortable. ${ }^{3}$ Its classic tasks comprise Atmosphere Control \& Supply (ACS), pressure control, relief and valves, monitoring of the air composition, Ventilation \& Air-Conditioning (VAC), Air Revitalization (AR) \& trace contaminant removal, Water Recovery \& Management (WRM), Fire Detection \& Suppression (FDS), and EVAs. The ColumBus ECLSS is designed to provide a healthy and comfortable work environment for up to three crew members and only covers VAC, atmosphere pressure control, and payload supply. It further supports the station-wide FDS system. The other ECLSS services like AR and WRM are provided by the host Space Station.

\section{A. Ventilation and Air-Conditioning}

As figure 5 exemplifies, the primary function of ColumBus ECLSS is VAC that comprises cabin air \& intermodule ventilation, air filtering, air temperature \& humidity control, and the monitoring of air composition. Furthermore, it provides air sampling support through a sample line to the Station AR system for trace contaminant control. Intermodule Ventilation (IMV) is maintained by the IMV Supply (ISFA) and Return Fan Assembly (IRFA). The fresh air supplied to the module flows through air ducts and passes the CHX first where it is cooled and dried. Then it is uniformly distributed to the inhabited area by two sets of four inlet diffusers in the two overhead standoff sections of the cabin. Before the air enters the CHX it is filtered by an High Efficiency Particulate Assembly (HEPA). The same amount of incoming air $\left(\approx 250 \mathrm{~m}^{3} / \mathrm{h}\right)$ has to be returned by the IRFA to the US laboratories for further processing. The used air is sucked through the Return Grid Sensor Housing (RGSH) and through a return air duct before it exits the module. In order to have sufficient air circulation in the inhabited cabin, the volume flow must be further increased by a recirculating or Cabin Fan Assembly (CFA) to $450 \mathrm{~m}^{3} / \mathrm{h}$. Columbus uses a pair of redundant Air Flow Sensors (AFS) to check if sufficient air flow is in the cabin for smoke detection.

Figure 5. Principle layout of the ventilation and air-conditioning system for the Columbus module.

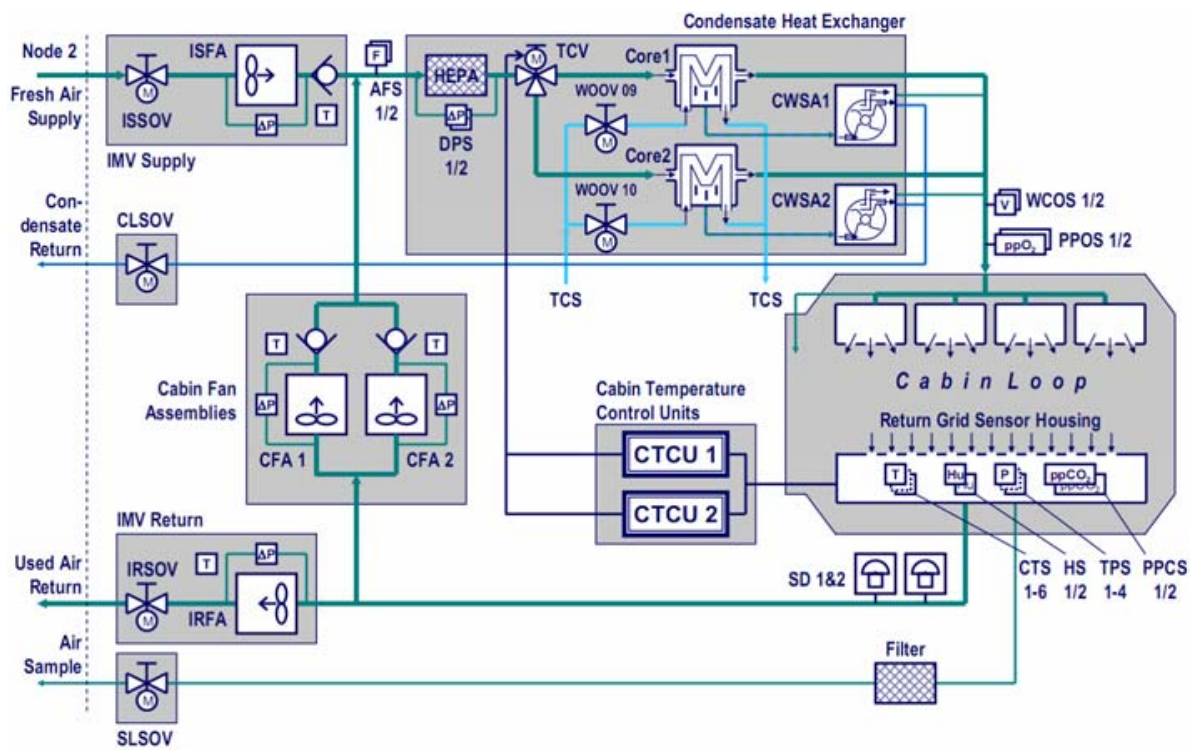

As already mentioned in section II B, the head loads are electrical equipment and the crew. Electrical equipment, however, only partially emits dissipative heat into the air while astronauts entirely radiate their body heat into the environment. So under nominal operational conditions there is always a gain of air heat in the cabin which makes air cooling necessary. Humidity sources are moist clothes or laundry drying in the cabin and the astronauts who exhale moisture and sweat. In order to keep the relative humidity within the operational limits of 30 and $70 \%$ and the cabin air temperature at a comfortable level of $23^{\circ} \mathrm{C}$ an airwater CHX is used which both cools and dries the air. It consist of a cooled transfer block or core and a Condensate Water Separator Assembly (CWSA). The second core in the second branch is not cooled and used as a warm bypass to mix the air streams. A Thermal Control Valve (TCV) directs the incoming air stream either more to the cold or more to the warm block depending on the need for cooling. The TCV is controlled by the main Cabin Temperature Control Unit (CTCU). It uses three Cabin Temperature Sensors 
(CTS) to measure air temperature and compares the average value against a set value. The coolant for the CHX is provided by the active TCS LTL. The coolant temperature must always be below the dew point temperature to force condensing on the hydrophilic-coated surfaces of the core. Because of micro-gravity conditions the liquid film must be sucked out off the block as a condensate-air mixture with the help of the CWSA that separates the water from the air by centrifugal force. The CWSA outlet pressure pushes the condensate via the condensate line to an USOS interface where it is further processed by the WRM system of the Space Station. A Water Carry-Over Sensor (WCOS) reacts whenever droplets are in the air stream. In contrast to temperature control there is no active control for humidity. Relative humidity is monitored by two redundant Humidity Sensors (HS) that are located in the RGSH. The entire VAC system can be isolated by Shut-Off Valves (SOV) in case of emergencies.

The monitoring of air composition in Columbus consists of the measurement of total, partial oxygen as well as partial carbon dioxide pressure. Four redundant Total Pressure Sensors (TPS) provide total pressure readings. Oxygen concentration is measured by two partial pressure $\mathrm{O}_{2}$ sensors (PPOS) and carbon dioxide concentration by two Partial Pressure $\mathrm{CO}_{2}$ Sensors (PPCS). Two redundant optical cabin Smoke Detectors (SD) are used to detect dust or smoke particles in the air. ISPR SDs are used to detect fire on payload level since the air volume in the rack is isolated from the cabin air.

\section{B. Pressure Control}

The second function of the Columbus ECLSS is pressure control that is only applied for off-nominal or contingency operations. Four redundant CDA Valves can be opened electrically in order to evacuate the module. This assembly has been designed for emergencies like toxic spill or fire but is not operationally in use. The Positive Pressure Relief Valves automatically blow air to the outside as soon as inside pressure of $103.4 \mathrm{kPa}$ is exceeded. The Negative Pressure Relief Valves are not used anymore and screwed with sealing caps to avoid possible leakage. They were required should the outside pressure exceed the inner pressure by more than 26 to $33 \mathrm{kPa}$, e.g., during the transportation from Bremen, Germany, to the Kennedy Space Center, Florida.

\section{Payload Supply}

The last function of the Columbus ECLSS is payload supply that covers vacuum, venting and nitrogen distribution. The $\mathrm{N}_{2}$ itself is, however, provided by the USOS which is illustrated by figure 6 .

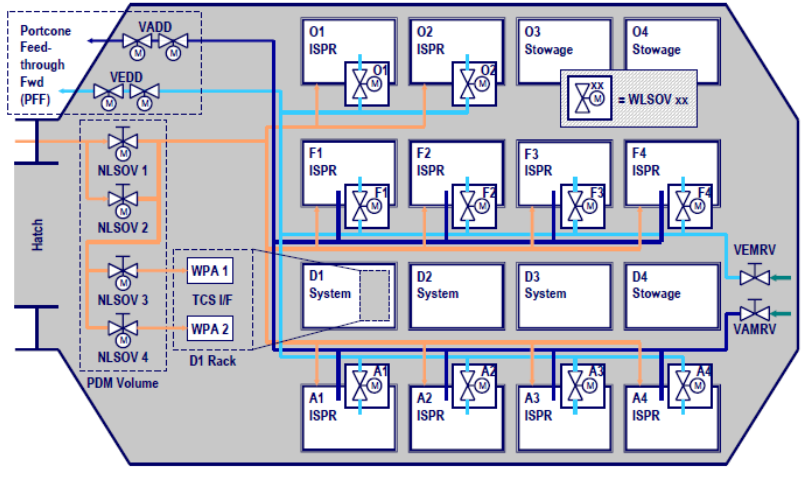

Figure 6. Payload supply architecture of Columbus ECLSS.

Vacuum Manual Return Valve (VAMRV) after VADD valves closure.

Nitrogen Line SOVs (NLSOV) are used for two different purposes: To provide $\mathrm{N}_{2}$ to the ISPRs and to the WPAs for accumulator re-pressurization. Two Venting Dump Device (VEDDs) valves are opened when waste gas must be dumped into outer space. Before doing so, Waste Line SOVs (WLSOV) must be opened. After closing all WLSOVs and the two VEDD valves, the venting line is re-pressurized with cabin air by manually opening the Venting Manual Return Valve (VEMRV). The four forward and the four aft racks are additionally equipped with vacuum lines to provide vacuum conditions to payloads. To do so two Vacuum Dump Device (VADDs) valves are opened to dump the air in the vacuum line to the outside. The vacuum line is re-pressurized in the same way as the venting line, i.e., opening the

\section{Orbit Anomaly Handling Process}

$\mathrm{T}_{\mathrm{n}}^{\mathrm{H}}$ HE Columbus Control Center has three major task: first, to monitor the orbital system (the ColumBus module), second to coordinate the European experiments and payloads on the ISS, and third to operate the ground communication infrastructure with the communication connections (lines) to all partners. ${ }^{4}$ The first two tasks are covered by the ColumBus Flight Control Team (FCT) that utilizes a mission control room 
for real-time operations execution. The control room is equipped with consoles displaying the telemetry data of the module in various formats to the FCT and allow access to the data and video channels of the ISS.

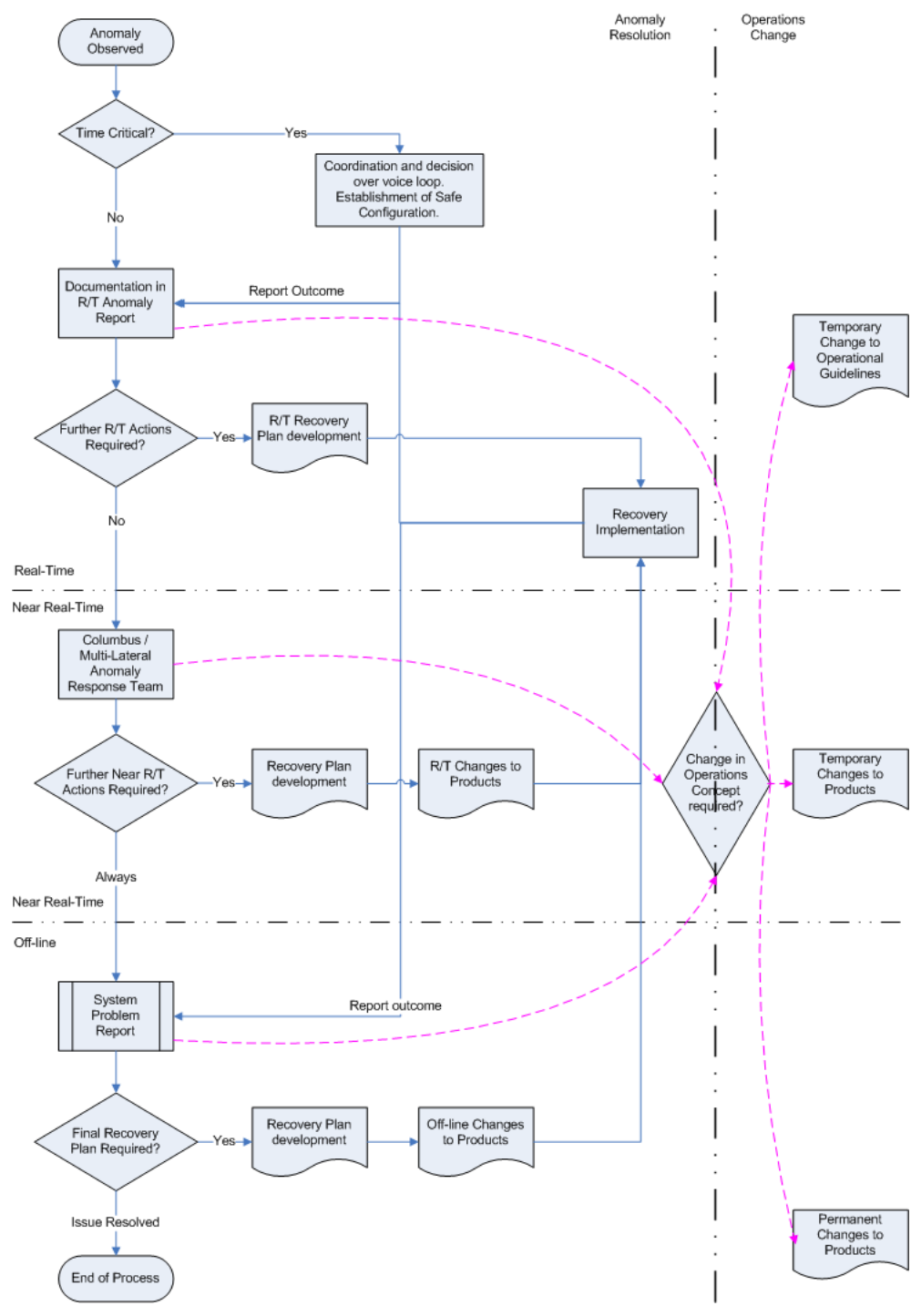

Figure 7. Anomaly resolution steps for real-time troubleshooting, workaround and final recovery plan development.
The FCT consists of Flight Controllers (FC) with dedicated task assignments. ECLSS and TCS are, e.g., covered by the Columbus Systems (COLSYS) position. During the mission the console positions are coordinated by a Flight Director (FD) who is responsible for the overall control of operations. The FD collects the relevant information from all team members and decides on the further course of action. He is in direct contact with the FDs in Houston and Moscow and with the Ground Control Team, and informs ESA managers about all events in the Columbus module.

In case of any observed anomaly, the FCT is requested to react according to a developed anomaly handling process that is documented in a so-called Joint Operational Interface Procedure. The process defines the actions to be taken in case of an anomaly to restore a safe condition and/or to regain operational status after a recovery procedure or plan. The process covers anomalies on Columbus system and payloads (ESA, US and other partners) but does not consider anomalies affecting ESA Payloads in the US Lab and elsewhere onboard the ISS.

Figure 7 illustrates the ColumBUS anomaly handling process flowchart that was developed for anomaly resolution and for possible change of operations concepts including modifications of guidelines and products. This resolution process ist devided in three sections and aims at finding a Real-Time $(\mathrm{R} / \mathrm{T})$, near

$\mathrm{R} / \mathrm{T}$ or workaround and a final recovery solution. In the following, we explain the resolution process stepwise.

1. The process is started when an on anomaly is observed. The FC or another person informs the FD about the observed anomaly.

2. If time is critical or a $\mathrm{R} / \mathrm{T}$ action is required, the $\mathrm{FC}$ proposes a real-time recovery or saving action (e.g., changing to a redundant system or otherwise). Safety relevant situation coordination and decision is done over the voice loop under the lead of the FD who will approve the recovery or saving action or give further instructions. The FC then implements the recovery or saving action, verifies its success and reports the outcome in anomaly report template of the Columbus flight note system.

3. A new R/T Anomaly Report is raised by the FC after a safe situation has been achieved. The report should include a proposed $\mathrm{R} / \mathrm{T}$ recovery solution.

4. The FD decides if further $\mathrm{R} / \mathrm{T}$ action is required. The $\mathrm{R} / \mathrm{T}$ recovery plan is developed and implemented by the FCT that verifies its success. The FD evaluates the anomaly and already decides if changes to the operations concept are required. 
5. All identified anomalies must be pre-screened in near R/T by the Columbus Engineering Support Team (COLEST) and other respective technical authorities. COLEST convenes a Multi-lateral or Columbus Anomaly Resolution Team (ART).

6. ART investigates and decides if further near $\mathrm{R} / \mathrm{T}$ actions and changes in the operations concept are required. If so ART dispositions a workaround plan that usually includes near $\mathrm{R} / \mathrm{T}$ changes of operational products provided by the FCT. The workaround or recovery plan is implemented by the FCT. COLEST documents the outcomes in the anomaly response section of the R/T Anomaly Report. The FD reviews the evaluations and decides if an additional revision of the near $\mathrm{R} / \mathrm{T}$ recovery plan is needed. ART always opens a System Problem Report (SPR) for the ESA Mission Resolution Board (MRB).

7. ESA MRB investigates and decides if further investigations and resolution of the anomaly as well as changes to the operations concept are required. If so MRB proposes a solution in the anomaly resolution section and dispositions a final recovery plan. Based on ART or MRB decisions products are being developed by the FCT that also implements the final recovery plan. The FD reviews the outcome and decides if an additional revision of the plan is needed.

8. ART and MRB decide if temporary changes to operational guidelines and products or permanent changes to products are needed. If so FCT changes guidelines and products accordingly.

9. The FD closes the R/T Anomaly Report when all issues are resolved and nominal operational status has been regained.

\section{Low Condensing Mode}

$\mathrm{T}^{\mathrm{N}}$ may 2008, ECLSS counterparts at the National Aeronautics and Space Administration (NASA) requested Iin their Mission Action Request System to raise the LTL CHX inlet temperature to a level that limits or even terminates condensate collection in Columbus. The rationale for this request was that American and Russian ECLSS counterparts have agreed to collect as much condensate as possible on the Russian Service Module (SM). In addition, this would decrease the difficulty of managing a wet/dry cycle of the USOS heat exchanger that is affected by the Columbus CHX collecting about $2 \mathrm{~L}$ condensate per day. This has resulted in more crew time being needed to off-load the USOS condensate tank and transfer water in canister to the $\mathrm{SM}$ for processing. COLEST immediately proposed in the response section to set this action request on hold until an ESA MRB approval has been given and all necessary actions for the implementation have been defined. The essential problem was that the Columbus CHX was not designed for a non-condensing mode, which implies that a technical workaround had to be developed with the need to introduce a new operational concept.

In June 2008, the ART agreed on slightly increasing the CHX inlet temperature to $7^{\circ} \mathrm{C}$ which reduces the amount of condensation in Columbus for about $50 \%$. Hence the need for periodic canister transfer was not eliminated but its frequency was reduced. This was declared as an interim solution since no change of automatic onboard control procedures were necessary and no CHX degradation could occur. The alternative option, increasing the inlet temperature to $10^{\circ} \mathrm{C}$ to completely stop condensation, would have implied the adaption of those procedures and a potential degradation of the CHX coating. However, for major air loop reconfigurations, e.g. a CHX dry-out, the transition back to nominal condensing mode was still required to collet enough condensate. COL FD and COL-SYS concurred to this interim solution and new operational products started being developed off-line based on the outcomes. New ECLSS and TCS procedures were developed and a new flight rule documented the new operational concept. To coordinate ESA and NASA activities related to this issue a new and so-called Operational Interface Procedure had to be written. NASA, e.g., always requests Columbus to go to nominal condensing mode for the duration of shuttle missions to collect condensate for further water processing. Finally, at the beginning of October 2008, the COLSYS group implemented the new operational products and successfully performed the transitions to Low Condensing Mode.

The increased CHX inlet temperature of $7^{\circ} \mathrm{C}$ is very close to the dew point temperature bringing the CHX into a low or quasi non-condensing condition. Since the forced condensation has nearly stopped the relative 
humidity starts to increase which raises the dew point temperature according to the following relation:

$$
\vartheta_{d}(\varphi, \vartheta)=\frac{241.2 \cdot \ln \varphi+\frac{4222.03716 \cdot \vartheta}{241.2+\ln \vartheta}}{17.5043-\ln \varphi-\frac{17.5043 \cdot \vartheta}{241.2+\ln \vartheta}}
$$

The CHX starts to condensate again until a new equilibrium between dew point spread (that is $\vartheta-\vartheta_{d}$ ) and condensate collection is reached. The interim solution thus increases the mean air humidity in the cabin slightly while the mean condensate collection in the CHX is lowered. Figure 8 shows this thermodynamical behavior by means of a recently performed CHX dry-out at the beginning of 2010 .

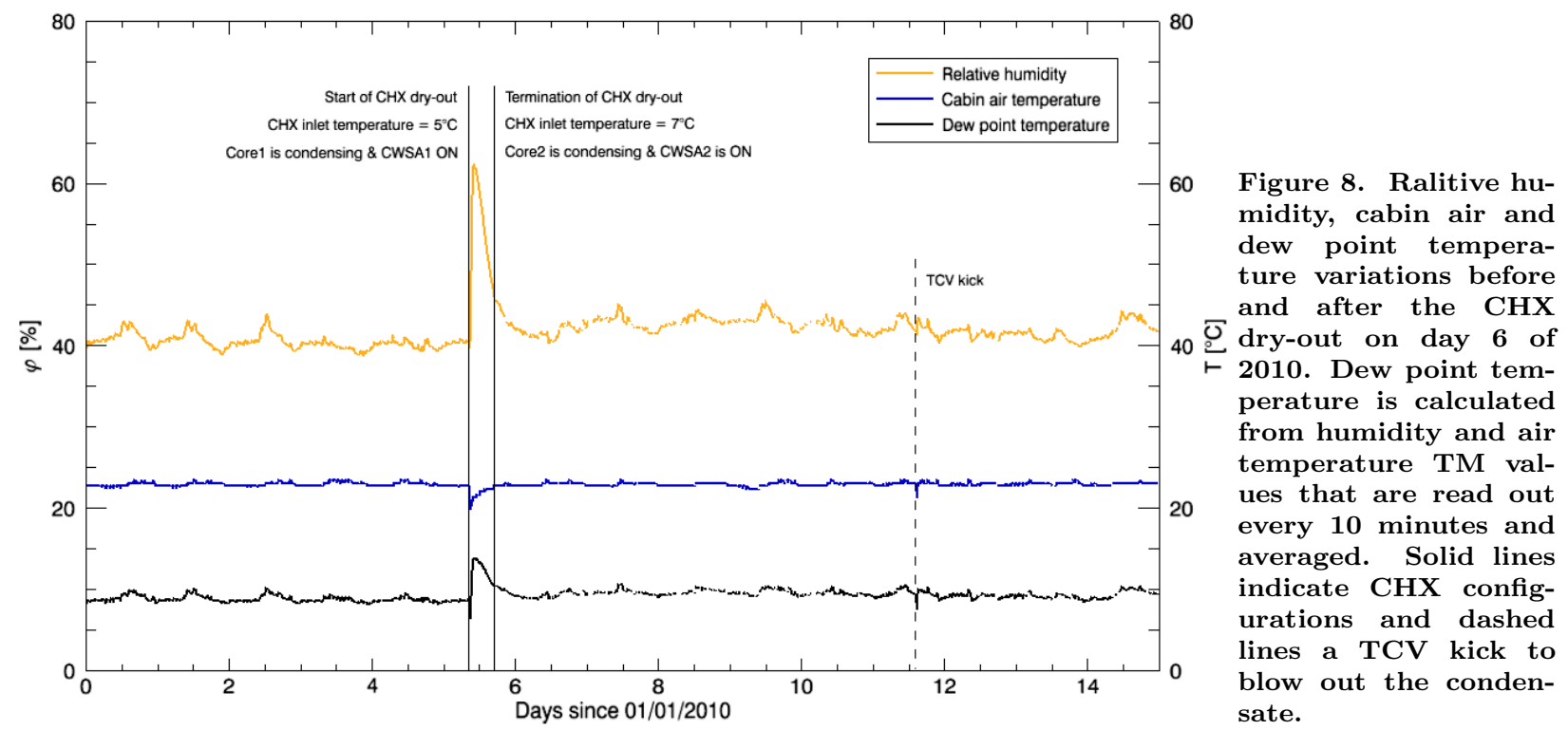

A complete dry-out of the cold core has to be performed every 6 weeks to prevent microbial and fungal growth on the hydrophilic coating. According to the new flight rule, a transition to nominal condensing mode has to be done one week before CHX dry-out start. As can be seen, the mean dew point temperature raises from $\approx 8.5$ to $10^{\circ} \mathrm{C}$ and the mean humidity from $\approx 41$ to $43^{\circ} \mathrm{C}$. Unfortunately, it cannot be proved if this humidity rise yields the intended reduction of condensate collection of $1 \mathrm{~L} /$ day since no condensate line flow sensors are available neither in Columbus nor in Node 2. At the beginning of the dry-out, both CWSAs or on and the TCV moves to a $50 \%$ position to direct the half of the air stream through the cold core causing a sudden drop of cabin air temperature. After CHX re-configuration, i.e., the cold core has become the warm and the former warm the cold core, the TCV sweeps to a $100 \%$ position to direct the entire air stream through the warm or former cold core. The coolant inside the drying core is still cold and not circulating and the surface gradually reaches ambient temperature. The air gets saturated with the condensate causing the positive humidity spike. After a certain time, the TCV moves slowly back to its control position to maintain a constant air temperature of $23^{\circ} \mathrm{C}$ and to start condensation on the cold core that was the former bypass core. The initial TCV position has been changed several times during operations to avoid a too strong drop of air temperature. After 8 hours, the dry-out process is terminated by deactivation of the former running CWSA and a transition to Low Condensing Mode. Figure 8 also shows the daily variation of humidity caused by crew activities. Around midnight, when nobody is in the module to work, the humidity reaches its minimum whilst around noon when the most crew activities are on-going it reaches its maximum.

\section{Smoke Detector Contamination}

$A^{\mathrm{T}}$ the end of 2008, a divergence between SD1 and SD2 obscuration and scatter values was observed A by COL-SYS. SD2 was degrading faster than SD1. Since the values were still in acceptable ranges no real-time troubleshooting was necessary to regain safe operational conditions. COL-SYS, however, raised an R/T Anomaly Report. He concluded that both SDs get dirty, SD2 faster than SD1 and recommended to monitor SD contamination behavior for long-term analysis. ART started investigations on R/T impacts and 
recommended the use of the percent trip parameter for evaluation purposes according to the smoke detector management flight rule. Since no near R/T solution could be found how to overcome the contamination problem ART raised an SPR to trigger an ESA MRB off-line investigation. It should be confirmed that this anomaly is related to optical contamination and not to another problem. Furthermore, the reason for the divergence of performance in both SDs was still unclear.

The dimensionless percent trip parameter is a calculated value of SD scatter and obscuration readings:

$$
\tau_{\text {trip }}=\frac{U_{\text {Sc. }}}{U_{\text {Sc., threshold }}}
$$

with the voltage scatter threshold

$$
U_{\text {Sc., threshold }}=\alpha_{\text {Sc.,trip }} \frac{U_{\text {Obsc. }}+4}{8} \quad \alpha_{\text {Sc.,trip }}=2.0
$$

that is the level the scatter signal must exceed in order to trip the detector and to trigger a fire alarm. Smoke detection in the ISS is thus based on a light scattering principle with two different sensors. An obscuration photo diode monitors the laser beam for attenuation and a scatter diode detects the stray light. The light is attenuated and scattered by particles passing the laser beam. The scatter trip variable permits a convenient method to correct the scatter threshold at any time. To account for detector degradation effects and optical contaminations the control Software (S/W) automatically evaluates the signal levels and lowers the scatter threshold accordingly. So if the obscuration dropped, e.g., to $3.0 \mathrm{~V}$ over time, the new scatter threshold would be $1.75 \mathrm{~V}$. The increased sensitivity thus offsets the degradation of the laser detection. When the obscuration signal, however, falls to $0 \mathrm{~V}$ (or $50 \%$ contamination) or less of its original level, the compensation technique is no longer possible. According to the SD management flight rule, the SDs have to be considered dirty when the $40 \%$-level is breached. After excess of the 50\%-level the monitoring of the SD must be inhibited to prevent a station-wide false fire alarm.

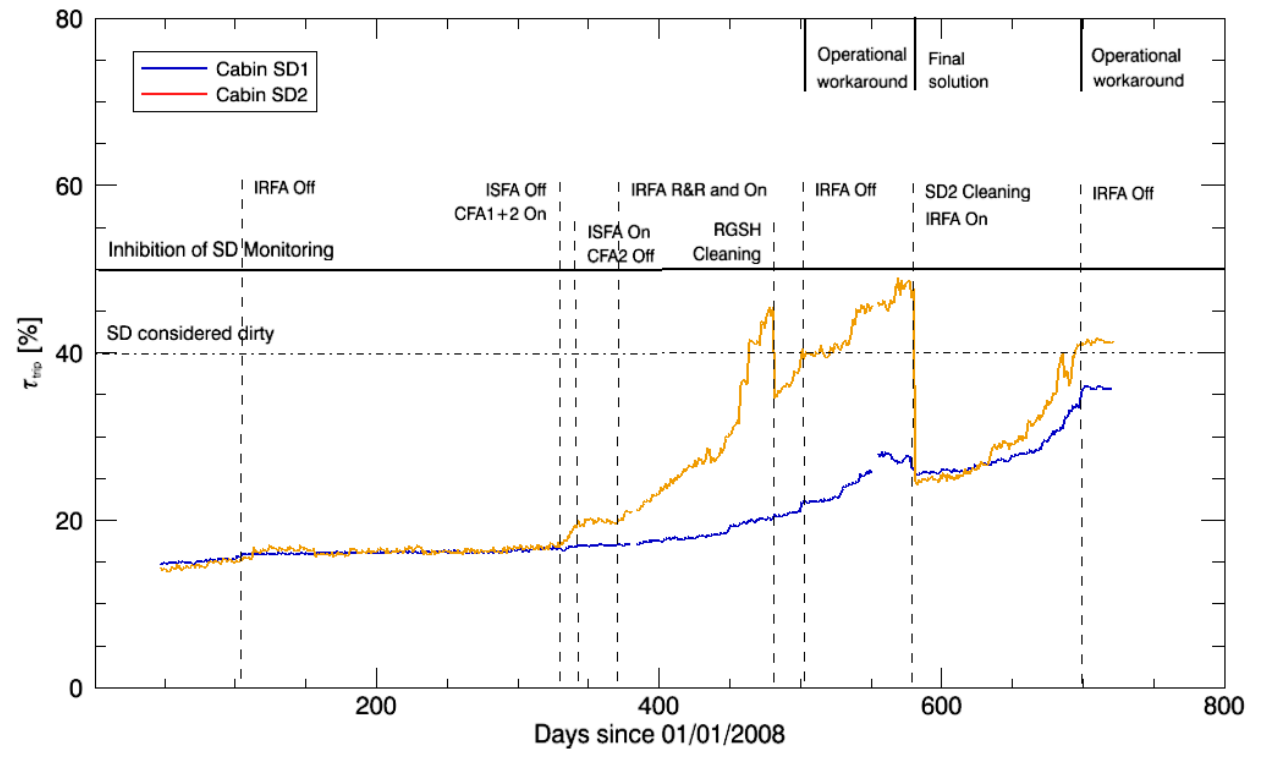

Figure 9. Trend analysis of percent trip calculated from SD obscuration and scatter readings. One value has been taken every day between 23:00 and 24:00 GMT for each SD. Dashed lines indicate airloop reconfigurations.

In order to further analyze SD performance behavior ART and ESA MRB proposed to perform airloop reconfigurations. Since the IRFA had already failed (April 2008) the ISFA was switched off in November 2008. To be compliant with an ECLSS flight rule and to have enough air flow for smoke detection the CFA2 was turned on afterwards which increased the air flow in the return air ducts from about 200 to $400 \mathrm{~m}^{3} / \mathrm{h}$. A much stronger divergence in performance was observed and a jump of about 16 to $20 \%$ of SD2 percent trip occurred, while the SD1 percent trip remained at 16\% (see figure 9). The resolution team confirmed optical contamination of both SDs and concluded that the location of the detectors is likely to be the reason for performance divergence. SD2 is mounted near to the RGSH, whereas SD1 is installed more downstream to the IRFA. In December 2008, the ISFA was switched on and CFA2 off which reduced again the air flow in the return air duct from 400 to $200 \mathrm{~m}^{3} / \mathrm{h}$. The SD2 performance trend was halted at a level of $20 \%$. In 
January 2009, however, the IRFA was replaced and activated again bringing the VAC system back to its nominal configuration. The sudden jump of airflow in the return air duct from 200 to $450 \mathrm{~m}^{3} / \mathrm{h}$ caused a rapid raise of SD2 percent trip whilst SD1 was only slightly degrading.

To avoid a breach of the monitoring inhibition level the resolution team started further investigations on how to access and clean SDs in the return air duct. A final recovery plan was developed, which also involved the development of new operational products: Crew and ground support procedure were designed in conjunction with flowcharts. New derived telemetry values like the percent trip of both cabin and all ISPR SDs were implemented and alphanumeric displays were adapted accordingly. Special telemetry displays were designed to monitor the cleaning process. Furthermore, special onboard hardware was required like $\mathrm{N}_{2}$ bottles which were provided by the USOS.

A regularly performed cleaning of the RGSH by a vacuum cleaner suddenly dropped the SD2 percent trip from 45 to $35 \%$ in April 2009 while the performance of SD1 was not affected. A part of the optical contamination was obviously removed by the RGSH cleaning and it was concluded that the pollution of the return grid might be linked to SD2 contamination. To mitigate the re-increasing SD2 contamination and to get more time for final recovery procedure development the COL-SYS group decided to turn off again the IRFA in oder to reduce the airflow in the return air duct. This was a typical operational workaround and could be declared as a temporary recovery solution. At the end of July 2009, the SD2 was finally cleaned by the crew which dropped the SD2 percent trip from $\approx 48$ to $25 \%$. The performance of SD1 also improved slightly as shown in figure 9. After switching on the IRFA, safe and nominal operational conditions were regained so that COL FD could close the anomaly report. The anomaly resolution process was completed. As expected both SD percent trips started to increase again and at the end of 2009 the operational workaround was re-applied to mitigate the contamination trend.

\section{Conclusions}

$\mathrm{T}^{\mathrm{T}}$ has been shown that the Columbus orbit anomaly handling process is not only useful for the troulbleshooting of $\mathrm{R} / \mathrm{T}$ anomalies but also a guideline for the resolution and development process of new operations concepts initiated by a mission action or change request. In the latter case, the resolution process directly starts in step 7 .

As far as the introduction of the Low Condensing Mode is concerned, it can be concluded that NASA and ESA understandings of requirements are still different regarding condensation removal despite previously agreed interface control documents. The Columbus CHX interactions with the Station environment and vice versa are stronger than expected and not completely analyzed. The CWSA behavior after mode transition, e.g., was still unclear and it took more than a year until it was declared nominal. The installation of additional new water processing assemblies in the USOS are still affecting CoLUmBUS operations. A failure in these assemblies, e.g. has evoked Columbus to stop moving condensate to Node 2 for some hours. For the design of future VAC systems in manned space vehicles or stations with several modules we therefore recommend the following:

- Humidity removal should be more configurable or even automized including total stop of condensation

- Station with multiple modules should have a more integrated humidity removal concept. In future planning of stations or vehicles with multiple modules this should be planned ahead

- Flow sensors in the condensate lines would give better insight into actual removed condensate quantity

- Intermediate storage capability in module with filling status would give more flexibility and could compensate failures in the main water processor assemblies.

To improve Columbus VAC system and to account for configurable and automized condensate removal we recommend to extend the CHX inlet temperature control law by humidity control, i.e., the LTL set point should be changed according to the measured humidity. We can imagine an adjustable LTL temperature control range between 5 and 7 or even $9^{\circ} \mathrm{C}$ combined with configurable set points for dry-out or non-condensing conditions. If the humidity drops below $30 \%$ the LTL temperature set point should be automatically raised by $2^{\circ} \mathrm{C}$ and if the humidity exceeds $70 \%$ the set point should be lowered again by $2^{\circ} \mathrm{C}$. This solution, however, encounters the following problems which have to be solved for possible ColumBus VAC system re-design: 
- The critical LTL and MTL control temperatures have to be adapted accordingly which implies the change of the automated onboard control procedures

- Modification of the control S/W in the electronic control units of both WPAs

- Accounting for uncertainties in the measurement of the total cabin humidity which distorts dew point calculation and LTL temperature control (e.g., HS1 and 2 are not measuring the humidity coming from Node 2)

SD contamination was not expected to happen so early in the operational phase. Especially the strong divergence in performance of SD2 with respect to SD1 revealed that optical contamination depends on SD location and is influenced by other air-polluting system equipment. The location of the SDs hampered the accessibility to the sensors and thus their cleaning. Before successful cleaning of SD2, complications during installation occurred, which shows the need for improvement of the installation process and/or the change of the sensor housing design. Furthermore, the development of the cleaning procedure required many efforts by the entire FCT, the engineering support and the ISS crew reducing the available time for research and other activities. For future human space flight missions, we therefore recommend the following:

- Placing the SDs in an air duct location where an easy access is ensured, e.g., by an air duct with a trap that can easily be opened somewhere in the cabin

- Simplification of the clamp mechanism. These simplifications will allow to develop cleaning procedures even before start of mission and reduce the time amount for maintenance.

- For planing purposes, a temporal extrapolation of the percent trip trend and thus the estimation of the time window can be made in which the SD should be cleaned or replaced within the 40 and $50 \%$ range of percent trip. The performance of SD1 shows the natural degradation of the sensor and the polynomial characteristic of the trend curve.

As a workaround fo Columbus we suggest to install an anti-aerosol mesh or filter somewhere upstream of the SDs. A mesh that is fine enough to filter small dust and other polluting particles but coarse enough to let pass the tiny smoke particles. This would even simplify the entire cleaning process and enhance the performance of both cabin SDs. COLEST is currently investigating a similar solution.

\section{A. Glossary}

$\begin{array}{llll}\text { ACS } & \text { Atmosphere Control \& Supply } & \text { MDPS } & \text { Micrometeorid \& Debris Protect. System } \\ \text { AFS } & \text { Air Flow Sensor } & \text { MLI } & \text { Multi-Layer Insulation } \\ \text { AR } & \text { Air Revitalization } & \text { MRB } & \text { Mission Resolution Board } \\ \text { ART } & \text { Anomaly Resolution Team } & \text { MTHX } & \text { Medium Temperature HX } \\ \text { CDA } & \text { Cabin Depressurization Assembly } & \text { MTL } & \text { Medium Temperature Loop } \\ \text { CFA } & \text { Cabin Fan Assembly } & \text { NASA } & \text { National Aeron. \& Space Administration } \\ \text { CHX } & \text { Condensate HX } & \text { NLSOV } & \text { Nitrogen Line SOV } \\ \text { CLSOV } & \text { Condensate Line SOV } & \text { PDM } & \text { Port Deck Module } \\ \text { COL } & \text { Columbus } & \text { PPCS } & \text { Partial Pressure } \mathrm{CO}_{2} \text { Sensor } \\ \text { COLEST COL Engenineering Support Team } & \text { PPOS } & \text { Partial Pressure } \mathrm{O}_{2} \text { Sensor } \\ \text { CTCU } & \text { Cabin Temperature Control Unit } & \text { RGSH } & \text { Return Grid Sensor Housing } \\ \text { CTS } & \text { Cabin Temperature Sensor } & \text { R/T } & \text { Real-time } \\ \text { CWSA } & \text { Condensate Water Seperator Assembly } & \text { SD } & \text { Smoke Detector } \\ \text { DPS } & \text { Delta Pressure Sensor } & \text { SLSOV } & \text { Sample Line SOV } \\ \text { DPSB } & \text { DPS Block } & \text { SM } & \text { Service Module } \\ \text { EADS } & \text { European Aeron. \& Defense Systems } & \text { SOV } & \text { Shut-Off Valve } \\ \text { ECLSS } & \text { Env. Control \& Live Support System } & \text { SPR } & \text { System Problem Report } \\ \text { ESA } & \text { European Space Agency } & \text { STS } & \text { Space Transportation System } \\ \text { ETCS } & \text { External TCS } & \text { S/W } & \text { Software } \\ \text { EVA } & \text { Extra Vehicular Activity } & \text { SYS } & \text { Systems } \\ \text { FC } & \text { Flight Controller } & \text { TCS } & \text { Thermal Control System } \\ \text { FCT } & \text { Flight Control Team } & \text { TCV } & \text { Thermal Control Valve }\end{array}$




$\begin{array}{ll}\text { FD } & \text { Flight Director } \\ \text { FDS } & \text { Fire Detection \& Suppression } \\ \text { GMT } & \text { Greenwhich Mean Time } \\ \text { HCU } & \text { Heater Control Unit } \\ \text { HEPA } & \text { High Efficiency Particulate Assembly } \\ \text { HS } & \text { Humidity sensor } \\ \text { HX } & \text { Heat Exchanger } \\ \text { I/F } & \text { Interface } \\ \text { IDL } & \text { Interactive Data Language } \\ \text { IFHX } & \text { I/F Heat Exchanger } \\ \text { IMV } & \text { Intermodule Ventilation } \\ \text { IRFA } & \text { IMV Return Fan Assembly } \\ \text { IRSOV } & \text { IMV Return SOV } \\ \text { ISFA } & \text { IMV Supply Fan Assembly } \\ \text { ISPR } & \text { International Standard Payload Rack } \\ \text { ISS } & \text { International Space Station } \\ \text { ISSOV } & \text { IMV Supply SOV } \\ \text { LTHX } & \text { Low Temperature HX } \\ \text { LTL } & \text { Low Temperature Loop }\end{array}$

$\begin{array}{ll}\text { TM } & \text { Telemetry } \\ \text { TPS } & \text { Total Pressure Sensor } \\ \text { US } & \text { United States } \\ \text { USOS } & \text { US Orbit Segment } \\ \text { VAC } & \text { Ventilation \& Air-Conditioning } \\ \text { VADD } & \text { Vacuum Dumping Device } \\ \text { VAMRV } & \text { Vacuum Manual Return Valve } \\ \text { VEDD } & \text { Venting Dumping Device } \\ \text { VEMRV } & \text { Venting Manual Return Valve } \\ \text { WCOS } & \text { Water Carry-Over Sensor } \\ \text { WFSV } & \text { Water Flow Selection Valve } \\ \text { WLSOV } & \text { Waste Line SOV } \\ \text { WMV } & \text { Water Modulating Valve } \\ \text { WOOV } & \text { Water On-Off Valve } \\ \text { WPA } & \text { Water Pump Assembly } \\ \text { WRM } & \text { Water Recovery Management } \\ \text { WTSB } & \text { Wet Temperature Sensor Block } \\ \text { CBM } & \text { Common Berthing Mechanism }\end{array}$

\section{Acknowledgments}

The authors would like to thank Dieter Sabath and Alexander Nitsch from the Columbus Mission Operations Service Project Management for their valuable comments and for providing figures 1 to 6 for which ESA holds the copyright. The plots in figures 8 and 9 have been created with IDL 7.0. The first author gratefully acknowledges the license sponsored by Jürgen Schwarz from the CREASO company in Wessling, Germany.

\section{References}

\footnotetext{
${ }^{1}$ Kohlhase, A. O. and Schlitt, R., Thermal Control. In: Handbook of Space Technology, Wiley \& Sons, Ltd, 2009

${ }^{2}$ Rojas, F., Analysis of Columbus Systems On-Orbit Performance by Processing and Evaluation of Telemetry Data, Diploma Thesis RT- DA-2009/03, Technical University of Munich, 2009

${ }^{3}$ Raatschen, W., Life Support Systems. In: Handbook of Space Technology, Wiley \& Sons, Ltd, 2009 2009

${ }^{4}$ Sabath, D. and Kuch, T., Operations for Human Space Flight. In: Handbook of Space Technology, Wiley \& Sons, Ltd,
} 